\title{
Germanica
}

\section{Extremes Essen und Körperinszenierungen: Thomas Bernhards Die Billigesser (1980) und Marco Ferreris La Grande Bouffe (1973)}

La représentation de grands mangeurs et de leurs corps: Les-Mange-pas-cher de Thomas Bernhard et La Grande Bouffe de Marco Ferreri

Extreme Eating and Representations of the Body: Thomas Bernhard's The Cheap Eaters (1980) and Marco Ferreri's La Grande Bouffe (1973)

\section{Martina Kopf}

\section{OpenEdition}

Journals

Édition électronique

URL : http://journals.openedition.org/germanica/3111

DOI : 10.4000/germanica.3111

ISSN : 2107-0784

Éditeur

Université de Lille

Édition imprimée

Date de publication : 30 décembre 2015

Pagination : 193-206

ISBN : 9782913857360

ISSN : 0984-2632

\section{Référence électronique}

Martina Kopf, «Extremes Essen und Körperinszenierungen: Thomas Bernhards Die Billigesser (1980) und Marco Ferreris La Grande Bouffe (1973) », Germanica [Online], 57 | 2015, Online erschienen am: 30 Dezember 2017, abgerufen am 06 Oktober 2020. URL : http://journals.openedition.org/germanica/ 3111 ; DOI : https://doi.org/10.4000/germanica.3111 


\title{
Extremes Essen und Körperinszenierungen: Thomas Bernhards Die Billigesser (1980) und Marco Ferreris La Grande Bouffe (1973)
}

\author{
Martina KOPF \\ Johannes Gutenberg - Universität Mainz
}

In der Überflussgesellschaft treten verschiedene Essverhalten nebeneinander, die nicht selten Auskunft über Lebenshaltungen, Einstellungen und Werte geben können. Wie auch bestimmte Essverhalten, so haben Lebensmittel einen symbolischen Gehalt ${ }^{1}$. Roland Barthes beschreibt in „Pour une psycho-sociologie de l'alimentation contemporaine“ (1961) Ernährung als Kommunikationssystem: „Qu'est-ce que la nourriture? Ce n'est pas seulement une collection de produits, justiciables d'études statistiques ou diététiques. C'est aussi et en même temps un système de communication, un corps d'images, un protocole d'usages, de situa-

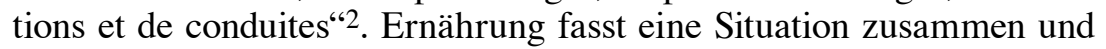

1. - Auf Sartres Feststellung, dass jede Nahrung ein Symbol sei, wird in der Kulinaristik-Forschung gerne verwiesen. Vgl. Simone de Beauvoir: La Cérémonie des adieux, Paris, Gallimard, 1981, S. 421.

2. - Roland Barthes: „Pour une psycho-sociologie de l'alimentation contemporaine“, in: Annales. Économies, Sociétés, Civilisations, 1961, 16e année, N. 5, S. $977-$ 986, S. 979. 
übermittelt diese, es handelt sich also um Zeichen, um eine funktionelle Einheit einer Kommunikationsstruktur. Diese Sinnstiftung entsteht nicht auf der Ebene der Erzeugung der Nahrungsmittel, sondern auf dem Niveau ihrer Umwandlung und ihres Verbrauchs ${ }^{3}$. Der italienische Regisseur Marco Ferreri hat einen ähnlichen Gedanken formuliert: „La nourriture, la consommation, si vous préférez, permet de tout saisir de notre société" 4 .

Der komparatistisch angelegte Beitrag möchte dem Essverhalten und Körperinszenierungen anhand von Thomas Bernhards Erzählung Die Billigesser (1980) und Marco Ferreris provokantem Film La Grande Bouffe (1973) nachgehen. Beide Werke thematisieren extreme Formen des Essens, die einen Bruch mit einem konventionellen Essverhalten darstellen. Im Mittelpunkt von Thomas Bernhards Erzählung stehen vier sogenannte „Billigesser“, die sich von Montag bis Freitag in der „Wiener Öffentlichen Küche“ (,WÖK“) ${ }^{5}$ zum jeweils billigsten Essen am „Billigessertisch“6 treffen. In Marco Ferreris Film, der bei den Filmfestspielen in Cannes 1973 für einen Skandal sorgte ${ }^{7}$, versammelt sich ein lebensmüdes Herrenquartett der Pariser Bourgeoisie zu einem kollektiven Überfressen, das keiner der vier Protagonisten überlebt. Das Askese und Sparsamkeit implizierende Billigessen stellt einen Gegenpol zum Überdruss assoziierenden Überfressen dar.

In einem ersten Schritt sollen diese Formen von Essverhalten und ihr symbolischer Gehalt analysiert werden, um in einem zweiten Schritt den Zusammenhang zwischen Essen und Körperlichkeit in beiden Werken aufzudecken.

\section{Extremes Essen I: Billigessen als Kategorie gesellschaftlicher Trennung}

Thomas Bernhards Erzählung Die Billigesser rückt das Unternehmen des Protagonisten Koller ins Zentrum, der vor 16 Jahren, ironischerweise

3. - Vgl. ebd., S. 981.

4. - Gérard Pangon; Jean-François Rauger (Hgg.): La Grande Bouffe, Paris, arte Éditions, 1997, S. 50-51.

5. - Vgl. zur Geschichte der „WÖK“, der „Wiener Öffentlichen Küche“, die nach dem Ersten Weltkrieg ins Leben gerufen wurde und eine bedeutsame Institution in der sozial orientierten Gastronomie darstellte: Thomas Bernhard: Werke (hg. von Martin Huber und Wendelin Schmidt-Dengler), Band 13: Erzählungen III (hg. von Hans Höller und Manfred Mittermayer), Frankfurt, Suhrkamp, 2008, S. 328.

6. - Thomas Bernhard: Die Billigesser, Frankfurt, Suhrkamp, 1980, S. 35. Im Folgenden (Bi).

7. - Vgl. Gérard Pangon: „Un parfum de scandale“, in: La Grande Bouffe, a.a.O., S. 9-22. Der Film wurde als pornografisches und skatologisches Werk bezeichnet. Vgl. Jean-François Rauger: „La Grande Bouffe, ou le cinéma de l'obsession“, in: La Grande Bouffe, a.a.O., S. 23-49, S. 24. 
durch einen Hundebiss, sein Bein verloren hat und seitdem an einer Studie über Physiognomik ${ }^{8}$ arbeitet. Untersuchungsobjekt sind die vier „Billigesser“, der Kaufmann Weninger, der Buchhändler Goldschmidt, der Magazineur Grill und der Professor Einzig, deren Physiognomie Koller typologisch beschreiben möchte. Aus dem Billigessen in der Kantine ist ein habitualisierter und ritualisierter Vorgang geworden, wie Koller feststellt:

„Zweifellos seien sie, das wäre sein erster Gedanke gewesen, schon immer darauf spezialisiert gewesen, das jeweils billigste Essen in der WÖK zu essen und in diesem Gedanken habe er auch schon wie selbstverständlich diese Leute an dem Ecktisch als die Billigesser bezeichnet und so waren sie von allem Anfang an für ihn immer schon die Billigesser gewesen, sie hatten immer das billigste Essen gegessen, das es in der WÖK gegeben hatte, er, Koller, habe sich nicht getäuscht, immer grundsätzlich das allerbilligste WÖKessen gegessen, solange er die WÖK aufgesucht habe und sie hätten, genauso wie er, niemals und unter keinen Umständen jemals eine andere als die billigste Essenskategorie gewählt, war es ihnen doch wie in der WÖK üblich freigestanden, immer zwischen vier Essenskategorien zu wählen“ (Bi, 34).

Es bleibt allerdings unklar, was das Billigessen, außer der Wahl der jeweils billigsten Essenskategorie in der „WÖK“, meint. Mit keinem Wort wird erwähnt, welche Gerichte die Billigesser zu sich nehmen. Billig zu essen bedeutet nicht, schlecht zu essen, wie man vermuten hätte können:

„Jahrelang war er [Koller] mit den Billigessern zusammen gewesen und hatte mit den Billigessern billig gegessen, so billig mit den Billigessern gegessen wie nirgends sonst und tatsächlich wie nirgends so billig und gut gegessen, denn in der WÖK habe er, Koller, immer billig und gut gegessen und nirgends hätte er jemals noch billiger und besser essen können“ ( $\mathrm{Bi}, 19)$.

Der günstige Preis wird der Qualität des Essens voran gestellt, wobei sich letztere erst durch das Billigsein zu konstituieren scheint. Gut ist das Essen nur, weil es billig ist. Angenommen das Essen wäre teurer, wäre es dann immer noch gut? Das Billigessen als Thema per se wird fragwürdig, denn dass Kollers Untersuchungsgegenstand ausgerechnet die Billigesser sind, wird als purer Zufall deklariert:

8. - Bernhard lässt seinen Protagonisten Koller Bezug auf das Werk Rudolf Kassners (1873-1959) nehmen, der ab 1908 Schriften zur Physiognomik verfasste. Während der Mensch in der aristokratischen Ständegesellschaft noch über ein Gesicht verfügte, hätte der moderne Massenmensch sein Gesicht verloren. Vgl. Thomas Bernhard: Werke (hg. von Martin Huber und Wendelin Schmidt-Dengler), Band 13: Erzählungen III (hg. von Hans Höller und Manfred Mittermayer), Frankfurt, Suhrkamp, 2008, S. 335. 
„[W]enn er, so Koller, an dem in Frage stehenden Tage zur alten Esche gegangen wäre, wäre er möglicherweise nicht auf die Billigesser gekommen, sondern auf etwas ganz anderes, wie er in jedem Falle, [...] auf ein anderes, möglicherweise sogar entgegengesetztes Thema gekommen wäre, auf ein vollkommen anderes, so er, als das, auf das er gekommen sei“" $(\mathrm{Bi}, 12)$.

Dass Koller der Blick auf die Billigesser plötzlich wie der „Blick in das Zentrum seiner Physiognomik, von welcher er sich nichts weniger als die Erfüllung seiner Lebensaufgabe versprochen habe“" $(\mathrm{Bi}, 13)$ erscheint, zeugt von Bernhardscher Ironie 9.

Da das Billigessen nicht weiter definiert werden kann, ist es bei Bernhard weniger als ernsthafter Ausdruck von Askese und Sparsamkeit zu verstehen, vielmehr handelt es sich um eine entleerte Vokabel, deren subversives Potenzial sich vor allem in den für Bernhard charakteristischen Wiederholungen manifestiert. Damit lässt sich das Billigessen nicht mehr als einfaches Zeichen eines Kommunikationssystems im Bartheschen Sinne dechiffrieren, doch kann ihm die Funktion einer Kategorie und damit einer Trennung zugesprochen werden. Am Tisch der Billigesser, einer geschlossenen Gesellschaft, findet der körperlich behinderte Koller einen Platz. Das Billigessen verbindet also, stiftet oberflächlich Gemeinschaft, doch Gemeinschaft ist bei Bernhard kaum möglich. Vielmehr kommt es zu der für ihn typischen „Zertrennung organischer Ganzheit, die zum polemischen Anschauungsunterricht der gesellschaftlichen Grundverhältnisse wird" 10 . Vor allem der Geistesmensch Koller ist zum Alleinsein verdammt: „Er war von Natur aus dazu angelegt gewesen, einen Geistesweg zu gehen, wie er es selbst nannte und das bedeutete nichts anderes, als dass er vollkommen allein zu gehen hatte" $(\mathrm{Bi}, 61)$.

Mit der Kategorisierung geht zugleich ein Exklusionsverfahren einher. Nur der Umstand, dass Koller ebenfalls ein „eingefleischter Billigesser" ist, verhindert, dass die Billigesser ihn von ihrem Tisch vertreiben $(\mathrm{Bi}, 35)$. Sie machen ihm , auf die angenehmste und tatsächlich zuvorkommendste Weise Platz" (Bi, 21) und haben Verständnis für seine Lage als Träger eines Kunstbeins, während Koller alle anderen Tische für seine Lage unpassend erscheinen: „Die Öffentlichkeit hatte auf diese Tatsache und also auf sein Kunstbein auf die niederträchtigste

9. - Als „Ironie-Werk“ wurde Bernhards Erzählung bezeichnet. Vgl. Franz Norbert Mennemeier: „Poetische Reflexion und Ironie. Zu Thomas Bernhards Prosawerk Die Billigesser", in: In Sachen Thomas Bernhard (hg. von Kurt Bartsch, Dietmar Goltschnigg, Gerhard Melzer), Königstein, Athenäum, 1983, S. 158-167, S. 162.

10. - Hans Höller: „,Es darf nichts Ganzes geben“, und ,In meinen Büchern ist alles künstlich'. Eine Rekonstruktion des Gesellschaftsbilds von Thomas Bernhard aus der Form der Sprache“, in: Bernhard. Annäherungen (hg. von Manfred Jurgensen), Bern/ München, Francke, 1981, S. 45-63, S. 57. 
Weise reagiert, indem sie so reagiert hatte, wie sie zu allen Zeiten den Krüppeln gegenüber reagiert hat" (Bi, 24). Der Billigesser-Tisch stellt damit eine eigene Tischordnung dar, die sich von den anderen Tischen in der WÖK absetzt. Nicht jeder hat eine Berechtigung, sich zum Typus des Billigessers zu zählen, wie der Erzähler festhält: „Ich selbst war immer ein WÖKaufsucher, aber niemals ein totaler Billigesser gewesen, weil ich nicht und niemals grundsätzlich billig gegessen habe, auch nicht in der WÖK und ich hatte keinerlei Berechtigung, mich jemals einen Billigesser nennen zu dürfen“ (Bi, 49). Der Erzähler, ein alter Freund Kollers und ein regelmäßiger Besucher der Gaststätte „Auge Gottes" lässt sich dort von Koller von seinem Projekt zur Physiognomie berichten. Menschen werden ihrem Gaststättenbesuch entsprechend in bestimmte Kategorien von „Menschengruppen“ (Bi, 102) eingeteilt, die über einen bestimmten Grad an Geistigkeit verfügen:

„Die WÖKmenschen hatte er [Koller] immer am höchsten eingeschätzt und sie immer auf die höchste Menschenstufe gestellt, die Augegottesmenschen und die Zögernitzmenschen waren von ihm jahrelang nurmehr noch verachtet gewesen. Und selbst von den WÖKmenschen hatte er sich schließlich getrennt und von ihnen nurmehr noch die Billigesser anerkannt. [...] Ehrlich gesagt, so Koller, sind die WÖKmenschen noch die charakterfestesten, die Augegottesmenschen die gemeinsten und die Zögernitzmenschen die niederträchtigsten“" (Bi, 114).

Dabei ist ein Wechsel vom einen Typus zum anderen durchaus möglich. Für Koller, ursprünglich ein Besucher des Café Zögernitz, wo im Unterschied zur WÖK „Geist-Atmosphäre“ (Bi, 107) herrschte, hat es Jahre gedauert bis er sich entschlossen hatte, ,das Zögernitz und also die Zögernitzmenschen aufzugeben und sich mit den WÖKmenschen und also mit den Billigessern zufrieden zu geben“ (Bi, 108). Die Wahl eines bestimmten Restaurants, welches wiederum für ein spezifisches Essverhalten steht, aber kaum näher beschrieben wird, generiert also einen eigenen Menschentypus. Doch diese Kategorisierung bleibt hohl, da es bis auf das Essverhalten keine gemeinsamen verbindenden Elemente gibt, sie verbildlicht auf abstrakte Weise eine zerklüftete Gesellschaft, in der es keine Einheit geben kann.

\section{Extremes Essen II: Überfressen als Kritik an der Überflussgesellschaft}

Ebenfalls exklusiv zeigt sich ein bürgerliches Herrenquartett in La Grande Bouffe, nämlich als Gourmetzirkel. Ziel ist ein kollektives „Sich-zu-Tode-fressen“, eine in die Länge gezogene Henkersmahlzeit, in einer abgelegenen Villa im Pariser Außenbezirk. Das elegante Haus wurde von Philippes Vater gekauft, doch wollte seine Mutter dort 
aus einem vielsagenden Grund nicht wohnen, wie Philippe seinen Freunden bei der Ankunft erklärt: „Elle trouvait que c'était un endroit trop frivole"11. Tatsächlich wird die bürgerliche Villa, ausgestattet mit Ballettsaal, Bugatti und einem weitläufigen Park schließlich zu einem frivolen Ort, an dem Völlerei, Maßlosigkeit und Lüsternheit bis zum Tod praktiziert werden.

Die vier Protagonisten, der Restaurantbesitzer Ugo, der Jurist Philippe, der TV-Produzent Michel und der erotomanische Pilot Marcello, vertreten die Pariser Bourgeoisie wie vor allem an ihren Essgewohnheiten deutlich wird. Als sie die Villa erreichen, sind sie bereits „satt“, nämlich des Lebens überdrüssig.

Im Mittelpunkt steht die aufwendige Zubereitung verschiedener Spezialitäten durch den Restaurantbesitzer und Brillat-Savarin zitierenden Koch Ugo. Zum Frühstück serviert er Nieren in Burgunder und heiße Schokolade, es folgen aufwendige Fleischspezialitäten zwischen Pizza und Pasta sowie immer höhere hausgemachte Torten. Kaum haben die vier Freunde die Villa erreicht, bringt ein Lieferant verschiedene Tiere, die auf zeremoniell-theatralische Weise aus dem Kühlwagen getragen und präsentiert werden: „,Un ,farouche cochon sanglier“... [...], prêt à toutes les marinades les plus subtiles.' [...] ,Deux superbes chevreuils à l'œil doux... dont la chair a tous les parfums...' $[\ldots], \ldots$ de la forêt" (LGB, 33-34). Es folgen Perlhühner, die mit Körnern und Wacholderbeeren gefüttert wurden, Bressehühner, Stubenküken aus den Ardennen, das Innere eines Charolais-Rinds und schließlich Lämmer von den Salzwiesen des Mont-Saint-Michel, wobei der alte Chauffeur von Philippe repetitiv ausruft: „Ça, c'est de la bonne viande!“ Die Qualität des Fleischs wird durch die jeweilige Herkunft garantiert, die die Funktion eines Gütesiegels übernimmt. Bemerkenswert ist in diesem Zusammenhang, dass die Produkte ausschließlich aus französischen Regionen kommen. Regionale französische Produkte scheinen für den Gaumen der französischen Bourgeoisie die „crème de la crème“" zu sein. Qualitative Lebensmittel sind also abhängig von der Region, jede Region hat ihre Spezialitäten, aber auch die Nation gilt damit als Qualitätsmerkmal. Angeblich wurden im Film nur die allerbesten Produkte verwendet, darauf macht eine Bemerkung im Vorspann aufmerksam, die auf die Essenslieferung durch den Pariser Feinkostspezialisten Fauchon verweist. Dass es sich beim „guten Essen“ um eine französische Angelegenheit handelt, hat der Regisseur in einem Interview betont:

11. - Im Folgenden wird aus dem Drehbuch von Marco Ferreri und Rafael Azcona zitiert, das in der Zeitschrift L'Avant-Scène Cinéma, revue mensuelle, janvier 2006, n 548: La Grande Bouffe, S. 24-75 abgedruckt wurde. Marco Ferreri; Rafael Azcona: La Grande Bouffe, a.a.O., S. 30. Im Folgenden (LGB). 
„C'est un film auquel j'ai pensé en France [...]. J'ai pensé que le sujet n'était pas négligeable dans un pays comptant, je crois, cent mille restaurants. Le bien-être exprimé dans le fait de manger est un signe, un drapeau. Le grand drapeau, on le laisse à la maison, mais la bourgeoisie a quantité de petits drapeaux, et la bouffe est une chose importante, une chose très française" 12 .

Vor allem das Austernwettessen mit Champagner und erotischer Diashow, das die Zusammenkunft einleitet, lässt sich als Zeichen im Bartheschen Sinne dechiffrieren. Bemerkenswert ist, dass die lebend gegessene Auster zwar die niedrigste Form tierischen Lebens darstellt, aber Luxus symbolisiert. Außerdem wird ihr die Wirkung eines Aphrodisiakums zugesprochen und sie gilt als Synonym für die Vulva ${ }^{13}$.

Die Austern-Mahlzeit scheint die erotische Komponente der Zusammenkunft also bereits vorwegzunehmen: Das Herrenquartett wird um drei Prostituierte und um die vollschlanke Lehrerin Andréa ergänzt ${ }^{14}$. Das gemeinsame Schlemmen, serviert werden verschiedene Geflügel am Spieß und ein Spanferkel, gegessen wird auf sinnliche Weise mit den Händen, verwandelt sich letztlich in Orgien zwischen Tisch und Bett, die den Film zum Skandal machten. Die Ordnung des gemeinsamen Mahls am Tisch löst sich bald auf, Tabus werden gebrochen, gegessen wird die ganze Zeit, ob im Stehen oder Liegen, im Bett und in der Garage. Selbst ohrenbetäubende Verdauungsgeräusche können die Protagonisten nicht vom Essen abhalten. Essensreste bleiben liegen oder landen im Aquarium, wie die Prostituierte Danielle irritiert feststellt: „Qu'est-ce qu'il fait ce poulet, ici? Dans un aquarium?" Worauf Michel kryptisch antwortet: „C'est un ,poisson-poulet““ (LGB, 45).

Schließlich sind es die Prostituierten, die zur mahnenden Instanz werden und den Bruch mit der Tischordnung als Tabubruch kritisieren. Dies bringt allerdings den Austritt aller drei Prostituierten aus dem Gourmetzirkel mit sich. Nachdem sie sich die ganze Nacht übergeben musste, bereits dies lässt sich als Form der Rebellion gegen das Überfressen deuten, verlässt Rita als erste die Villa und Danielle bringt die Kritik schließlich auf den Punkt: „Vous êtes grotesques, grotesques et dégoûtants. [...] Pourquoi vous mangez si vous n'avez pas faim? C'est pas possible, c'est pas de la faim!“ (LGB, 54). Essen ohne Hunger zu haben, das Überfressen, scheint moralisch nicht vertretbar. Mit ihrer Aussage übt Danielle nicht nur Kritik an dem Unternehmen der vier Protagonisten, sondern kritisiert indirekt die Überflussgesellschaft,

12. - Jean-François Rauger: „La Grande Bouffe, ou le cinéma de l'obsession“, a.a.O., S. 36.

13. - Vgl. Ad de Vries: Dictionary of Symbols and Imagery, Amsterdam/London, North-Holland Publishing Company, 1974, S. 355.

14. - Für eine Analyse der Geschlechterrollen bietet der Film reichlich Material. 
in der unkontrolliertes Essen ein gängiges Essverhalten darzustellen scheint. Philippes Versuch, den übersättigten Michel zum Essen zu bringen, indem er ihn an das Hungerproblem in Indien erinnert, treibt die groteske Szene schließlich auf den Gipfel.

Interessanterweise brechen Schwierigkeiten im Nahrungsverhalten ,just in jenen Augenblicken auf [...], wo - in einer Überflussgesellschaft - Probleme der Nahrungsbeschaffung gelöst erscheinen und der Wohlstand zu herrschen beginnt"15. Das Unternehmen, ein radikales Überfressen bis zum Tod, spiegelt in überspitzter Weise das Essverhalten der Überflussgesellschaft wider und wird damit zur Karikatur derselben. Das allmähliche Versagen aller vier Herrenkörper durch ein Übermaß an Wollust und Völlerei lässt sich metaphorisch als Verfall einer genusssüchtigen Bourgeoisie deuten. Diese (Kapitalismus-) Kritik ließe sich heute natürlich auf verschiedene Gesellschaftsschichten übertragen.

\section{Extremes Essen und Körperinszenierungen}

Mit der Thematisierung extremen Essverhaltens gehen sowohl in Die Billigesser als auch in La Grande Bouffe die Inszenierung von Körper und die Darstellung körperlicher Bedürfnisse einher. Einer dualistischen Anthropologie zufolge wird der Körper vom Geist getrennt betrachtet:

„So erfindet die durch Platon und das Christentum entwickelte und von Kant fortgesetzte Metaphysik eine dualistische Anthropologie, die den Menschen in zwei ungleiche Seinsweisen zerteilt: in ein ,niedriges', sich ernährendes, sinnliches Wesen und in ein ,höheres' Wesen einer rein geistigen Vernunft. Mithilfe dieser metaphysischen Polarisierung konnte der diätmoralische Diskurs die körperliche Bedürfnisbefriedigung einem Bereich der biologischen Zwänge und geistlosen Naturnotwendigkeiten zuordnen" ${ }^{16}$.

Während der Park in Die Billigesser einen Ort des Geistes, nämlich des Nachdenkens, darstellt, wird mit dem Gasthaus als Welt des Organischen, und damit des Körpers, ein Gegenpol assoziiert ${ }^{17}$. In $\mathrm{La}$ Grande Bouffe wird die sinnliche Lust zum leitenden Prinzip erkoren.

Dem diätmoralischen Diskurs entsprechend werden Körper und das Körperliche und damit auch kulinarische Genüsse abgewertet. Wollust und Völlerei gelten als fleischliche Sünden und als Bedrohung für den menschlichen Geist: „Von je her sind es neben der triebhaften Sinnlichkeit

15. - Gerhard Neumann, ,Jede Nahrung ist ein Symbol“, a.a.O., S. 402.

16. - Harald Lemke: „Gastrosophische und philosophische Aspekte der Kulinaristik", in: Kulturthema Essen (hg. von Alois Wierlacher, Gerhard Neumann und Hans Jürgen Teuteberg), Berlin, Akademie Verlag, 1993, S. 147-156, S. 149.

17. - Vgl. Sahbi Thabet: „Mittagstischphilosophie. Zur Darstellung des Raumes in Thomas Bernhards Die Billigesser" , in: Etudes Germaniques, 2000, 4, S. 781-801, S. 788. 
der Sexualität gerade die kulinarischen Lüste des Essens (und Trinkens), in denen der klassische Rationalismus die größte Bedrohung einer vernunftbegabten Menschheit vermutet"18. La Grande Bouffe widmet sich genau dieser von der Diätmoral formulierten „Bedrohung“, nämlich der desaströsen Kombination von Völlerei und Wollust, der schließlich alle vier Herrenkörper intendiert zum Opfer fallen.

\section{Einverleibung, Wollust, Ausscheidung}

Essen und Körper stehen in Ferreris Film in einem engen Zusammenhang, der sich anhand zahlreicher Formen bemerkbar macht. Vollkommener kulinarischer Genuss scheint sich erst durch die Anreicherung von sexueller Lust völlig zu entfalten, dies erklärt die Einladung der Prostituierten. Zudem wird kulinarischer Genuss als sexuelle Lust interpretiert. So erklärt Philippe beim gemeinsamen Essen mit Andréa und den Prostituierten: „Je dis qu'une femme qui apprécie la nourriture..." (LGB, 47). Unterbrochen wird er in diesem Moment durch Marcello, ergänzt aber kurze Zeit später: „...qui fréquente n'importe qui“" (LGB, 48). Diese Ergänzung ließe sich sowohl auf den begonnen Satz zu dem Typus der das Essen schätzenden Frau anwenden, als auch auf den ebenfalls von Philippe geäußerten Satz über Marcello zu Andréa: „Excusez-le, c'est un pilote de ligne...“ (LGB, 48).

Die Präsenz der drei Prostituierten wird damit für die Fressorgie selbstverständlich. Das Motiv der nackten Frau zieht sich wie ein roter Faden durch die Villa, wo es sich in Form von Skulptur und Gemälden wiederspiegelt. Die üppige Andréa ${ }^{19}$ scheint die Maßlosigkeit der wollüstigen älteren Herren zu verkörpern, ihre voluminösen Hinterbacken dienen Ugo schließlich als Utensil bei der Herstellung der „Tarte Andréa“. Der Tabubruch in dieser Szene wird spätestens dann deutlich, wenn die Doppelfunktion des weiblichen Hinterns, als sexuelles Symbol, aber auch als Ausscheidungsorgan deutlich wird. Was als Appetit anregend gilt, kann ebenso Ekel erregen. Mit dieser Gratwanderung zwischen Genuss und Appetit sowie Ekel macht der Film auf die fließenden Grenzen aufmerksam.

Während Ugo beim Austernwettessen das weibliche Geschlechtsorgan mit einer Auster vergleicht (LGB, 37), dienen weibliche Brüste Andréa als Vorlage für einen Pudding, den sie Philippe, dem letzten Überlebenden des Herrenquartetts, als Henkersmahlzeit serviert. Ironischerweise stirbt er beim Verspeisen des Brust-Puddings, der sowohl Völlerei als auch Wollust symbolisiert.

18. - Harald Lemke: „Gastrosophische und philosophische Aspekte der Kulinaristik“, a.a.O., S. 150.

19. - Die Darstellerin Andréa Ferréol hatte für den Film zwanzig Kilo zugenommen. 
Die bei Marcello nach diversen Zusammenkünften mit den Prostituierten einsetzenden Ejakulationsprobleme, die als Konsequenz einer gleichermaßen kulinarischen aber auch sexuellen „Übersättigung“ gedeutet werden können, begründet Andréa bezeichnenderweise mit ihrer Leibesfülle: ,Je suis trop grosse“ (LGB, 64). Was an dieser Stelle ebenso als Mangel an sexueller Attraktivität gedeutet werden kann, verweist gleichzeitig auf den Topos der Übersättigung.

Allerdings rückt der Körper nicht nur als Generator sexueller Lust und als sexuelles Objekt in den Fokus. Mit der Einverleibung ist zugleich die Ausscheidung verbunden und damit die Thematisierung des Körpers als Verdauungsapparat. Es sind vor allem die überspitzt inszenierten körperlichen Bedürfnisse, die für einen Tabubruch sorgen.

Bereits bevor das große Fressen beginnt, imitiert Ugo während der Fleischlieferung Verdauungsgeräusche: „Il appuie la sentence d'un énorme bruit de pet avec sa langue" (LGB, 35). Damit nimmt er ein zentrales Motiv des Films vorweg. Mehrere Szenen widmen sich Michels Flatulenz, die er zu Beginn noch zu verbergen sucht, mit der Zeit aber für alle akustisch und olfaktorisch erlebbar macht. Michel erliegt schließlich dem Versagen seines Verdauungsapparates, wie die visuelle Aufbereitung deutlich macht: „Michel est assis au pied de la rambarde, mort, les bras ballants et les jambes écartées, au milieu desquelles s'est répandue und petite flaque d'excréments" (LGB, 70). Nach dem Motto „Friss oder stirb nicht“ (LGB, 56), drängen ihn seine lebensmüden, aber bis zum Ende heißhungrigen Freunde geradezu in den Tod. Trotz massiver Verdauungsprobleme wird Michel von ihnen dazu genötigt, das von Ugo zubereitete Kartoffelpüree zu essen. Ironischerweise wird es ihm als „purée médicale“ (LGB, 56) mit einer wohltuenden Wirkung angepriesen.

Die Ausscheidung scheint die Einverleibung schließlich zu dominieren. Das Versagen der Herrenkörper wird durch einen Kollaps der überbeanspruchten Sanitäranlagen vorweggenommen. Nach einem Rohrbruch im Badezimmer wird das Haus von Exkrementen überschwemmt. Dieser „Sintflut der Scheiße“20, die symbolisch als Untergang der Bourgeoisie und der Überflussgesellschaft gedeutet werden kann, werden die Protagonisten, auch olfaktorisch, nicht mehr Herr, wie Ugo feststellt: „L'odeur de la merde, elle ne nous quittera jamais!” (LGB, 66). Tatsächlich erinnert Philippe Ugos letzte Torte an Exkremente, wie er feststellt: „C'est de la merde“ (LGB, 71).

Ugo leitet schließlich seinen eigenen Tod ein, indem er die riesige Kuppeltorte aus Geflügelpastete und Brioche zubereitet und diese mit Eiern dekoriert, diese seien der jüdischen Tradition gemäß als

20. - Im französischen Drehbuch heißt es ,déluge universel“. Vgl. Marco Ferreri; Rafael Azcona: La Grande Bouffe, a.a.O., S. 66. 
Symbol des Todes zu deuten (LGB, 70). Da Andréa und Philippe die Nahrungsaufnahme verweigern, verspeist er sein kulinarisches Kunstwerk allein.

In Ugos Todeskampf findet die Symbiose von Völlerei und Wollust schließlich einen weiteren Höhepunkt: Auf dem Küchentisch liegend verspeist er seine Torte und bittet Andréa um eine Masturbation. Die Leiche Ugos bleibt aufgebahrt in seinem Domizil, der Küche, während die Leichen von Michel und Marcello im Kühlhaus einen Platz finden.

Die Essensreste, nämlich das frisch gelieferte Fleisch, geht schließlich regelrecht vor die Hunde - auf Andréas Wunsch wird es im Garten deponiert, wo sich die Hunde darüber her machen. Der Überfluss an hochwertigem Essen steht allegorisch für das Verhalten der Überflussgesellschaft und, auf das Individuum zurückgeworfen, metaphorisch für einen Lebensüberdruss. Nahrung, eigentlich eine Lebensnotwendigkeit, bewirkt durch ein extremes Essverhalten physische Destruktion.

\section{Physiognomik, Geistesmensch und Esslokal}

In Die Billigesser sind Essverhalten, Körper und Geist auf komplexe Weise miteinander verwoben. Bernhard scheint geradezu das Prinzip der Diätmoral, die Dualität von Körper und Geist, auf spielerische Weise aufzugreifen. Bereits die Tatsache, dass Koller die Physiognomie, in der es schließlich zu einem Rückschluss vom Körperlichen auf das Geistige kommt, der Billigesser erforschen möchte, verweist auf eine angenommene Korrespondenz zwischen Essverhalten, Körper und Geist:

„Die Physiognomien aller vier Billigesser seien, so Koller, grundlegend und folgerichtig von dem jahrzehntelangen WÖKbesuch der Billigesser geprägt gewesen, alle vier hätten sie vor jeder anderen eine von ihm, Koller, so genannte WÖKphysiognomie, also in erster Linie diese WÖKphysiognomie und erst in zweiter Linie ihre ganz persönliche eigene angeborene, unabhängig von der WÖK im Laufe ihres Lebens auf ihren Gesichtern gewachsene, unaufhörlich und tatsächlich ununterbrochen auf ihren Gesichtern von ihrer Geschichte und von der ganzen Welt- und Naturgeschichte verursachte“ $(\mathrm{Bi}, 101)$.

Im Sinne eines „Man ist, was man isst“ formt das Essen also nicht nur diejenige Körperregion, die wichtigster körperlicher Ausdruck jeden Individuums ist, das Gesicht, sondern formiert darüber hinaus soziale Gemeinschaft und stabilisiert Trennungen in Form von Kategorien innerhalb der Wiener Gesellschaft.

Der Rückschluss vom Essverhalten, und von der Kantine, auf das Körperliche und schließlich auf das Psychische wird ausgerechnet vom körperlich behinderten „Geistesmenschen“ Koller vorgenommen, der sich dem Billigessertisch erst nach seinem Unfall anschließt. Der 
Geistesmensch nimmt eine der Masse entgegen gestellte Position ein, er hat ,schon im Augenblick der Geburt den Kampf gegen die Masse aufzunehmen“ (Bi, 84), gleichzeitig ist Koller aber wegen seiner physiognomischen Studien auf die Gesellschaft angewiesen.

Just an dem Tag, an dem er mit einem Kunstbein aus dem Krankenhaus entlassen wird, ändert sich sein Leben:

„[D]enn es wäre ja auch kein Zufall gewesen, dass er sechzehn Jahre vorher gerade in dem Augenblick auf die Billigesser gestoßen sei, von welchem er heute sagen könne, dass er die eigentliche und also die entscheidende geistige Wende in seinem Leben herbeigeführt habe und zwar genau an dem Tage, an welchem er nach der damals schon siebzehn Wochen zurückliegenden Amputation seines Beines aus dem Wilhelminenspital entlassen und in seine Wohnung in der Krottenbachstraße zurückgekehrt war“" $(\mathrm{Bi}, 15)$.

Wie der Erzähler feststellt, leitet Koller geistige Stärke anhand von körperlicher Schwäche ab: „Die Tatsache, dass er von frühester Kindheit an augenleidend und dann, in der Reife, plötzlich auch noch ein Krüppel gewesen war, hatte ihm tatsächlich eine von ihm so bezeichnete höhere Geistesweihe und Geisteswürde verliehen“" (Bi, 54-55). Der Hundebiss - als geistige Wende - habe schließlich für eine geistige „Krönung“ (Bi, 56) Kollers gesorgt. Die wissenschaftliche Forschung, die Physiognomik, und damit sein geistiges Lebenswerk, verdanke er seinem „Lebensunglück“ (Bi, 15), nämlich dem Verlust eines Körperteils durch einen ironischerweise ,heißhungrigen“, bissigen Hund. Erst durch die Beeinträchtigung des Körpers kann es zu „Geisteskapital“ (Bi, 46) und schließlich zu „Geistestriumph“ (Bi, 48) kommen. Koller verdankt der WÖK, dem Ort der Billigesser und damit dem Arbeitsplatz seiner wissenschaftlichen Studie, ,seine Existenz und das heißt genauso seine Körperexistenz wie seine Geistesexistenz und vor allem seine Geistesexistenz" (Bi, 20). Zu einem Höhepunkt der Bernhardschen literarischen Kulinaristik, sowohl auf inhaltlicher als auch formaler Ebene, kommt es, indem Bernhard Körper und Geist als von der WÖK gleichermaßen abhängige Instanzen zusammenführt. Ohne die WÖK wäre Koller „längst körperlich und geistig verhungert“ (Bi, 20). Bewahrt das billige Essen in der WÖK vor einem körperlichen Hungertod, so die Zusammenkunft mit den Billigessern und die damit verbundene wissenschaftliche Betätigung als Forscher vor einem geistigen Aus. Die WÖK wird für Koller zur ,eigentlichen Lebensretterin und letztenendes Lebensbewahrerin“" $(\mathrm{Bi}, 20)$.

Doch eine Vernichtung des Körpers geschieht letztendlich nicht wie von Koller gewünscht - „seinem Geist zuliebe“ (Bi, 79), sondern das körperliche Versagen, der Sturz Kollers über sein Kunstbein im Stiegenhaus in der Krottenbachstraße, sorgt für eine totale 
Bewusstlosigkeit des Protagonisten, aus der er nicht mehr zu retten war. Das körperliche Versagen sorgt für ein geistiges Versagen. Nicht nur Kollers Geist ist damit ausgelöscht, auch sein Geistesprodukt Die Billigesser erhält keine Vollendung wie der letzte Satz der Erzählung besagt: „Die Billigesser waren verloren gewesen wie so viele Geistesprodukte, von welchen uns ihre Erfinder gesprochen haben“ (Bi, 150). Durch seinen abrupten Tod kann Koller sein Lebenswerk nicht mehr fertigstellen. Die - geistige - Selbstverwirklichung bleibt, wie so oft im Bernhardschen Werk, dem Protagonisten verwehrt, dessen Bemühungen grotesk wirken. Der Geistesmensch scheint dem „Körper“ Welt nicht gewachsen und wird von diesem vernichtet. Bernhards Erzählung liest sich vor dem Hintergrund einer ironischen Darstellung eines Geistesmenschen auch als „Karikatur des eigenen Schreibens“21.

Dem billig essenden Geistesmenschen stellt Bernhard einen nicht billig essenden, körperlich gesunden Geistesarmen als Kontrahenten gegenüber. Während Koller nach eigenen Worten eine geistige Entwicklung durchlaufen habe, sei die des Erzählers eine geistig entgegengesetzte Entwicklung (Bi, 58). Während die Kranken und Geistreichen ihre Mahlzeiten in der WÖK einnehmen, essen Gesunde und Geistesarme im Restaurant „Auge Gottes“. Der Erzähler entpuppt sich als Augegottesmensch, der im Unterschied zu Koller kein Geistesmensch ist, aber dessen Existenz ebenso vom regelmäßigen Restaurantbesuch abhängt: „Das Auge Gottes war lange Zeit das Lokal gewesen, ohne welches ich nicht existieren hätte können, wie er, Koller, ohne seine WÖK nicht existieren hätte können“ (Bi, 67). Als Koller mit dem Erzähler das „Auge Gottes“ besucht, trinkt er „unter allen diesen stumpfsinnigen, geistfeindlichen fleisch- und gemüsefressenden Augegottesmenschen, die er zurecht verachte" $(\mathrm{Bi}, 90)$ nur ein Glas Bier. Das „Auge Gottes“ bietet Koller nicht nur keine körperliche Sättigung, sondern zeigt sich ihm als unzuverlässiger Geistesort: „Auf einmal sei ihm doch klar geworden, dass es unmöglich sei, mir im Auge Gottes die Billigesser zu erklären, dazu sei ein absolut zuverlässiger Geistesort die Voraussetzung und ein solcher absolut zuverlässiger Geistesort sei das Auge Gottes in keinem Falle, was es ihm jetzt unmöglich mache, mir seine Billigesser vorzutragen" (Bi, 148). Nur im Wertheimsteinpark, dem ,einzigartigen und vollkommen störungsfreien Geistespark“ (Bi, 148) sei ein neuer Vortrag möglich.

Das in beiden Werken thematisierte extreme Essverhalten nimmt eine bestimmte Funktion ein: Während das Überfressen in La Grande Bouffe das Verhalten der Pariser Bourgeoisie der 70er Jahre kari-

21. - Eva Marquardt: Gegenrichtung. Entwicklungstendenzen in der Erzählprosa Thomas Bernhards, Tübingen, Niemeyer, 1990, S. 164. 
kiert und Kritik an der Überflussgesellschaft und möglicherweise deren Essverhalten übt, zeigt sich das Billigessen bei Bernhard als Kategorisierung, die auf eine zerklüftete Gesellschaft hindeutet. Das geschilderte Essverhalten impliziert also in beiden Fällen eine mehr oder weniger indirekte Gesellschaftskritik und übernimmt damit eine allegorisch-metaphorische Aufgabe. Durch den vorausgehenden Prätext, im Fall von Bernhard seine Vorstellung von Gesellschaft und im Fall von Ferreri die Gesellschaftskritik, liest sich das Überfressen als Allegorie auf das Verhalten der Überflussgesellschaft, während die Billigesser metaphorisch eine Trennung innerhalb der Gesellschaft verdeutlicht.

Die Thematisierung extremen Essverhaltens und die damit verbundene Kopplung an Gesellschaft bringen Körperinszenierungen verschiedener Art mit sich. Während Ferreri das Prinzip der Diätmoral in sein Gegenteil umkehrt, indem er körperlichen Bedürfnissen den Vorrang lässt, jongliert Bernhard mit dem Dualismus von Geist und Körper. Dies spiegelt sich auch in seiner Sprache wider, die mit dem Essensvokabular auf spielerische Weise verfährt. Nicht nur von einer körperlichen Sättigung oder einem körperlichen Verhungern ist die Rede, sondern ebenso von einer bzw. einem geistigen.

Damit zeigt sich, dass sich das in Literatur und Film dargestellte Essverhalten wesentlich komplexer gestaltet: Billigessen und Überfressen sind nicht auf Askese und Genuss reduzierbar, sondern werden in der künstlerischen Umsetzung mehrdeutig. Dabei wäre das zu beherzigen, was Wendelin Schmidt-Dengler zu Bernhards Texten unter Rückgriff auf Peter Szondi, dem es wiederum um das Lesen der Texte Paul Celans ging - formuliert hat:

„Es geht nicht darum, ,sich für eine der verschiedenen Bedeutungen zu entscheiden, sondern zu begreifen, dass sie nicht geschieden sind, sondern eins. Die Mehrdeutigkeit, Mittel der Erkenntnis geworden, macht die Einheit dessen sicher, was verschieden nur schien. Sie dient der Präzision“"22.

22. - Wendelin Schmidt-Dengler: „Elf Thesen zum Werk Thomas Bernhards“, in: Der Übertreibungskünstler. Studien zu Thomas Bernhard (hg. von ders.), Wien, Sonderzahl, 2., erweiterte Auflage, 1989, S. 107-112, S. 110. Schmidt-Dengler zitiert Peter Szondi: Celan-Studien, Frankfurt, Suhrkamp, 1971, S. 111. 